\title{
EIN BEITRAG ZUR THEORIE DES BLEISAMMLERS.
}

\author{
Von Prof. Dr. F. Foerster.
}

(Mitteilung aus dem anorganisch-chemischen Laboratorium der K. S. Technischen Hochschule zu Dresden.)

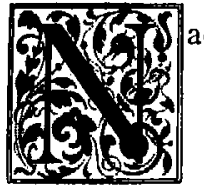

achdem durch die schönen Untersuchungen von Elbs und Schönherr die von Schoop verfochtene Darrieussche Theorie über die Vorgange im Bleisammler als den Thatsachen nicht ausreichend entsprechend erwiesen worden ist, besteht wohl, angesichts der von Streint $z^{1}$ ) nachgewiesenen Übereinstimmung der aus der Wärmetönung des Vorganges $\mathrm{PbO}_{2}+2 \mathrm{H}_{2} \mathrm{SO}_{4}+\mathrm{Pb}=2 \mathrm{~Pb} \mathrm{SO}_{4}+2 \mathrm{H}_{2} \mathrm{O}$ berechneten und der wirklich beobachteten elektromotorischen Kraft des Akkumulators und seines demzufolge fast verschwindenden Temperaturkoëffizienten, kaum noch ein Zweifel, dass im wesentlichen im Bleisammler bei der Entladung das Superoxyd an der Anode und das Blei an der Kathode in Bleisulfat übergehen, wahhrend dieses beim Laden an der Anode in Bleisuperoxyd, an der Kathode in Blei verwandelt wird. Die Untersuchung der Einzelpotentialsprünge an den Elektroden hat weiter ergeben, dass derjenige an der Kathode im Verhaltnis zu der hohen elektromotorischen Kraft des Bleisammlers gering, derjenige an der Anode aber die Hauptquelle derselben ist.

Es erheischt daher die Superoxydplatte, welche ja auch in technischer Hinsicht die mühevollsten und durch die nunmehr gelungene, ökonomisch durchführbare Formierung nach Planté gekrönten Arbeiten veranlasst hat, ein hervorragendes theoretisches Interesse. Dies ist nur noch gesteigert worden, dadurch dass $\mathrm{Haag}{ }^{2}$ ) nachgewiesen hat, dass die Zunahme des inneren Widerstandes eines Bleisammlers während der Entladung im wesentlichen durch das Auftreten eines Übergangswiderstandes an der Anode bedingt ist.

Der erste, welcher die Vorgänge an der Superoxydelektrode im Sinne unserer Ionentheorie eingehend erörterte, war Le Blanc ${ }^{3}$ ): welcher annahm, dass bei der Entladung von ihr Bleitetrahydroxyd, d. h. vierwertige Bleiionen nebst je vier Hydroxylionen in Lösung gingen, erstere hier aber nicht bestehen könnten, sondern unter Übergang in zweiwertige Bleiionen alsbald die Halfte ihrer Ladung an die Anode abgäben. Die năhere Betrachtung

1) Wien. Akad. Ber. 103, Jan. I 894.

2) Diese Zeitschr. 3, $42 \mathrm{I}$.

3) Lehrb, d. Elektrochemie, S. 222 u. ff. dieses Vorganges ergab eine einfache Erklärung der allmăhlichen Abnahme der elektromotorischen Kraft des Bleisammlers von 2,0 auf 1,8 Volt wăhrend der Entladung.

In wesentlichen Punkten hat sich Elbs ${ }^{4}$ ) der Le Blanc'schen Theorie angeschlossen. Es gelang ihm, unter bestimmten Bedingungen bei der Elektrolyse von Bleiacetat an der Anode das Acetat des vierwertigen Bleis $\mathrm{zu}$ erhalten, welches sich sehr leicht hydrolytisch in freie Essigsalure und Bleisuperoxyd spaltet. Demgemåss schloss er, dass im Akkumulator beim Laden vierwertige Bleiionen, d. h. Bleidisulfat $\mathrm{Pb}\left(\mathrm{SO}_{4}\right)_{2}$ in der Lösung entstände, welches alsbald in Bleisuperoxyd und freie Schwefelsäure hydrolytisch zerfiele. Das erstere wird sich dann ${ }^{5}$ ) in der von Le Blanc dargelegten Weise bei der Entladung bethatigen. Die letztere wird hiernach also nicht als völlige Umkehrung der Ladungsvorgange betrachtet; ist es doch auch bisher in der That nicht gelungen, die dem Akkumulator zugeführten Energiemengen vollständig aus ihm wieder zu gewinnen. So giebt also die Le BlancElbs'sche Betrachtungsweise Aufschluss über .zwei wichtige Eigenschaften des Bleisammlers.

Die Le Blan c'sche Anschauung hielt Liebenow ${ }^{6}$ ) für etwas willkürlich und stellte ihr gegenüber die Ansicht auf, dass in jeder Bleilösung, wenn auch in geringer Menge, neben $\stackrel{+}{\mathrm{Pb} \text {-ionen auch } \mathrm{Pb}} \overline{\mathrm{O}}_{2}^{-}$ ionen vorhanden seien, welche, ahnlich wie Metallionen ihre positiven Ladungen an der Kathode, so ihre negativen Ladungen an der Anode abgeben, und bei der Entladung des Akkumulators aus dem Superoxyd der Anode ăhnlich unmittelbar wie die Metallionen aus einer Metallelektrode wieder in die Lösung hineinwandern, eine Annahme, welche durch die verhăltnismässig hohe Leitfăhigkeit des Bleisuperoxyds gestützt wird. In der Lösung sollen dann die $\overline{P b} \bar{O}_{2}$-ionen sehr leicht im Sinne der Gleichung

$$
\overrightarrow{\mathrm{Pb}} \overline{\mathrm{O}}_{2}+{ }_{4} \stackrel{+}{\mathrm{H}}=\stackrel{+}{\mathrm{Pb}}+2 \mathrm{H}_{2} \mathrm{O}
$$

4) Diese Zeitschr. 3, 7 I u. ff.

5) Elbs scheint sich den Entladungsvorgang an der Anode ein wenig anders $z u$ denken.

6) Diese Zeitschr. 2, 420 u. 653 . 
zum grosssen Teil in positiv-zweiwertige Bleiionen übergehen. Liebenow folgert die Möglichkeit des Entstehens von $\overline{P b} \bar{O}_{2}$-ionen aus der Löslichkeit von Bleioxyd in Alkalien, wobei offenbar die Ionen $2 \stackrel{+}{K}$ und $\overrightarrow{P b} \bar{O}_{2}$ aus dem Salz $\mathrm{Pb}(O K)_{2}$ sich bilden; in der That zeigte sich, dass bei der Elektrolyse dieses Salzes das Blei im wesentlichen nach der Anode wandert. Daraus schliesst Liebenow, dass auch andere Bleisalze, z. B. Bleinitrat, in wasssriger Lösung nicht nur in gewöhnlicher Weise, sondern auch nach dem Schema: $2 \mathrm{~Pb} \mathrm{~N}_{2} \mathrm{O}_{6}+2 \mathrm{H}_{2} \mathrm{O}=\stackrel{+}{\mathrm{P} b}+{ }_{4} \stackrel{+}{\mathrm{H}}$ $+\overrightarrow{P b} \overline{O_{2}}+{ }_{4} \mathrm{NO}_{3}$ elektrolytisch dissociiert sind.

Dieser Auffassung ist Lö $\mathrm{b}^{7}$ ) beigetreten, welcher bei der Elektrolyse organischer Bleisalze stets mehr oder weniger Blei an der Kathode und Bleisuperoxyd an der Anode erhielt, wathrend die ursprünglich vorhandenen Säurereste unvermindert, nur unter Übergang in die betreffenden freien Säuren in der Lösung verblieben. Zur Erklärung hierfür nimmt er an, dass die fraglichen Bleisalze ausser der elektrolytischen auch eine hydrolytische Spaltung erfahren, und dass das durch die letzcere erzeugte Bleihydroxyd in die Ionen $\overrightarrow{P b} \overline{O_{2}}+2 \vec{H}$ zerfallt, von denen die der ersteren Art an der Anode zur Abscheidung gelangen. Die Auffassung, dass das Bleisuperoxyd bei der Elektrolyse aus $\overline{\mathrm{Pb}} \overline{\mathrm{O}}_{2}$-ionen entstehe, ist auch in die neueste Auflage von Classen's "Chemische Analyse durch Elektrolyse" ubernommen worden, ohne dass das Vorhandensein einer entgegengesetzten Ansicht auch nur angedeutet wäre. Mir scheint hierfür kein genügender Grund vorhanden zu sein, vielmehr die Le Blanc'sche Ansicht durchaus den Vorzug zu verdienen. Die Gründe hierfür will ich versuchen im Nachfolgenden darzuthun an der Hand von Betrachtungen, zu denen ich zuerst bei Gelegenheit einiger die Elektrolyse von Kupfersulfatlösungen betreffenden Beobachtungen geführt wurde.

Vor allem ist daran zu erinnern, dass die Annahme vierwertiger Bleiionen nichts Willkürliches an sich hat, dass vielmehr die Fahigkeit des Bleis, im Zustande der Vierwertigkeit aufzutreten, angesichts des Vorhandenseins von Bleitetraalkylverbindungen oder der bleisauren Salze schon lange nicht

7) Diese Zeitschr. 2, 495; 3, roo.

8) 4. Auflage, S. 19, 20, 45 . mehr zweifelhaft ist, und die Herstellung von Bleitetrachlorid, Bleitetrafluorid, Bleidisulfat, Bleitetrahydrophosphat, Bleitetraacetat und Bleitetrapropionat ${ }^{9}$ ) das Entstehen vierwertiger Bleiionen als möglich dargethan hat.

Was zunächst die Begründung der Theorie von Licbenow und Löb anlangt, so hat schon Elbs darauf hingewiesen, dass die von Löb dazu herangezogenen Beobachtungen an organischen Bleisalzen einfacher und ungezwungener durch die Annahme der Entstehung vierwertiger Bleiionen erklärt werden. Die Erscheinungen bei der Elektrolyse von Bleilösungen lassen sich im wesentlichen folgendermassen zusammenfassen: Wahrend zunächst an der Kathode $\stackrel{+}{P b \text {-ionen }}$ entladen werden, wandern die đquivalenten negativen Reste nach der Anode, wo ebensoviel $\stackrel{+}{\mathrm{Pb}}$ ionen vierwertig geworden sind und alsbald doppelt soviel negative Reste als vorher in der Lösung in $+++$

Anspruch nehmen. Die Konzentration der $P b$-ionen vermehrt sich bis zu einem von der Konzentration, Temperatur und anderen Eigenschaften des Elektrolyten gewiss nicht unabhängigen, immer aber nur geringen Masse, und alsdann erfolgt Hydrolyse des Salzes des vierwertigen Bleis unter Ausscheidung von Superoyd bezw. von dessen Hydrat an der Anode. Es entsteht dabei freie Săure, d. h. Wasserstoffionen, und diese werden nach der Kathode gelangen und hier je nach der obwaltenden Stromdichte und ihrer Konzentration neben mehr oder weniger Bleiionen entladen werden; nur wenn gleichviel Blei an der Kathode abgeschieden ist, wie an der Anode als Superoxyd gefallt wird, muss die Gasentwicklung an der Kathode aufhören. Sind von vornherein genügend Wasserstoffionen unter sonst geeigneten Bedingungen (salpetersaure Lösunng) anwesend, so kann die Abscheidung von metallischem Blei ganz unterbleiben, und alles Blei als Superoxyd an der Anode gefallt werden. Andererseits wird hier bei nicht zu hoher Stromdichte kein Superoxyd auftreten, wenn Anionen von so geringer Haftintensităt vorhanden sind (z. B. die der Oxalsaure), dass ihre Entladung einen geringeren Arbeitsaufwand erfordert als die Umwandlung zweiwertiger Bleiionen in vierwertige.

Aber auch die von Liebenow zur Stütze seiner

9) Litteratur: Ber, d. d. chem. Ges. 26 (1893), 1434. Zeitschr. f. anorgan. Chem. 4, 100, 747; 7, 1. Journ. Chem. Soc. 1892, II $36 ; 1894,393 ; 1896,212$. 
Ansichten angezogene Deutung des Verhaltens einer alkalischen Bleioxydlösung lässt manche Einwände zu, ganz abgesehen davon, dass, worauf Elbs schon hinwies, der Rückschluss auf andere als die an Hydroxylionen so reichen alkalischen Bleilösungen ja ein recht fragwürdiger ist. Es entsteht weiter die Frage: warum geben nicht auch Lösungen von Zinkoxyd oder Kupferoxyd in Kalilauge an der Anode Superoxyde, da sie ja doch auch sicherlich Ionen $\overline{Z n} \bar{O}_{2}$ bezw. $\overline{\mathrm{C}}_{\mathrm{O} \mathrm{O}_{2}}$ enthalten? Ferner fragt man, wie geht es zu, dass durch blosse Entionisirung das Ion $\bar{P} b \bar{O}_{2}$, in welchem doch offenbar das Blei zweiwertig ist, in das Bleisuperoxyd übergeht, in welchem man ja, da es stetiges Produkt der hydrolytischen Spaltung der Salze des vierwertigen Bleies ist, unzweifelhaft das Blei im Zustande der Vierwertigkeit annehmen muss?

Diese Schwierigkeiten lassen sich nun aber leicht heben, wenn man auch bei der Elektrolyse alkaliv scher Bleilösungen die Möglichkeit eines Überganges zweiwertiger Bleiionen in vierwertige ins Auge fasst, eine Möglichkeit, welche für $\stackrel{++}{Z n}$ - und $\stackrel{+}{C u \text {-ionen nicht }}$ besteht. ${ }^{10}$ ) In einer Lösung von Bleioxyd in Kalilauge finden sich aber ebenso sicher wie $\overrightarrow{P b} \vec{O}_{2}$-ionen auch $\stackrel{+}{P} b$-ionen in gewisser Konzentration vor; denn bei der Elektrolyse solcher Lösungen entsteht an der Kathode reichlich metallisches Blei. Gelangen nun die $\stackrel{+}{P b}$-ionen an die Anode, so können sie $++++$

hier in $P b$-ionen übergehen, worauf wieder sich zwi-

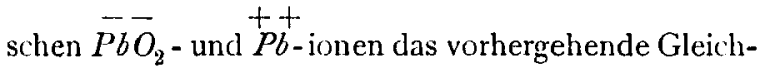
gewicht herstellen muss u. s. f. Die $\stackrel{++++}{P b \text {-ionen treffen }}$ aber mit einer grossen Zahl von Hydroxylionen zusammen, mit denen sie nach der Gleichung $\stackrel{++++}{P b}+\overline{O H}$ $=\overline{\mathrm{PbO}}_{3}+3 \mathrm{H}_{2} \mathrm{O}$ die negativen Ionen der Bleisalure geben, deren, Kalisalz dann, wie andere áhnliche Salze an der Anode unter Fortwandern des Alkalis die unlösliche freie Säure, bezw. deren Anhydrid, das Bleisuperoxyd abscheidet. $\mathrm{Da}$ in der in Rede stehenden alkalischen Bleioxydlösung offenbar die Konzentration der $\stackrel{+}{P} b$-ionen klein ist, treten die

10) Das bekannte bräunliche Kupferdioxyd ist ein Superoxyd vom Typus des Baryunsuperoxyds, da es mit Schwefelsäure Wasserstoffsuperoxyd giebt; Verbindungen eines vierwertigen Kupfers sind nicht bekannt. vorerwăhnten Erscheinungen nur bei niederen Stromdichten glatt auf, waihrend, wie Beetz ${ }^{11}$ ) fand, bei höheren Stromdichten sich dem Bleisuperoxyd reichlich Bleihydroxyd beimischt, welches infolge Verarmung der die Anode umgebenden Lösung an Alkali ebenso wie vorher das Bleisuperoxyd einer Hydrolyse seine Entstehung verdankt. Dass das Bleisuperoxyd eher als das Bleioxyd sich hierbei ausscheiden muss, folgt aus seiner gegenüber derjenigen des letzteren geringeren Löslichkeit in Kalilauge. Man überzeugt sich hiervon leicht, wenn man eine alkalische Bleioxydlösung mit Wasserstoffsuperoxyd versetzt; dabei fallen reichliche Mengen von Bleisuperoxyd aus.

Schliesslich aber ist es leicht, das Entstehen von vierwertigen Bleiionen an der Anode durch den Versuch darzuthun: Elektrolysiert man eine bei $0^{0}$ mit Bleichlorid gesättigte, mit etwas Salmiak versetzte starke Salzsảure bei $0^{0}$ mit etwa $0,5 \mathrm{Amp} . / \mathrm{qdm}$ Stromdichte mehrere Stunden zwischen Platinelektroden, so erhalt man an der Anode keine Spur Bleisuperoxyd, sondern es entsteht Bleitetrachlorid und bildet reichliche Mengen des dem Platinsalmiak ausserlich so ahnlichen und ihm in seiner Zusammensetzung entsprechenden Salzes $\left(\mathrm{NH}_{4}\right)_{2} \mathrm{PbCl}_{6}$ mit allen ihm von seinen Entdeckern zugeschriebenen Eigenschaften. ${ }^{12}$ ) Man thut bei dieser Elektrolyse gut, die Kathode mit Pergamentpapier zu umwickeln, da etwa herabfallender Bleischwamm das genannte gelbe Salz leicht in weisses Bleichlorid umwandelt: $\mathrm{PbCl}_{4}+\mathrm{Pb}=2 \mathrm{PbCl}_{2}$. Elektrolysiert man aber eine neutrale Bleichloridlösung bei $\mathrm{O}^{0}$, so entsteht auch hier neben freiem Chlor Bleitetrachlorid; dies kann sich aber nicht halten, sondern erleidet infolge der Abwesenheit freier Sazure alsbald Hydrolyse unter Abscheidung von Bleisuperoxyd, welches nach wenigen Sekunden beginnt, die Anode gleichmässig zu überziehen.

Aber selbst von all diesem abgesehen, lässt die Auffassung, dass an der Anode des Bleisammlers vierwertige Bleiionen entstehen, die Vorgånge in ihm mit einer grossen Klasse anderer elektrolytischer Erscheinungen unter einheitlichen Gesichtspunkten erscheinen.

Die Arbeit des elektrischen Stromes bei der Elektrolyse wässeriger Lösungen besteht ja an den

11) Pogn. Ann. 6r, 209.

12) Ein Herstellungsverfahren für dieses interessante Salz lässt sich hierauf wegen der geringen Löslichkeit des Bleidichlorids nicht begründen. 
Elektroden nicht nur in der Überführung von Elementen aus dem Ionenzustand in den nicht ionisierten Zustand, bezw. der Umkehrung dieses Vorgangs, sondern, allgemeiner gesprochen, in einer Veränderung der Ionenladungen. Bei Ionen wie die des Wasserstoffs, Zinks, Silbers und ahnlicher Elemente, von denen wir nur eine Verbindungsstufe kennen, besteht diese Änderung allerdings in nichts anderem als der völligen Ladung bezw. Entladung der Atome durch den Strom. Wir kennen aber eine grosse Zahl von Elementen, welche verschiedene Verbindungsstufen aufweisen, verschiedene Wertigkeit annehmen und daher Ionen mit verschiedenen elektrischen Ladungsmengen zu bilden vermögen. Bei der Elektrolyse der Lösungen solcher Metalle kann der Strom je nach seiner Dichte sehr verschiedene Wirkungen hervorbringen; er kann, statt wie gewöhnlich Sauerstoff zu entwickeln, an der Anode auf positiven Ionen von niederer Wertigkeit positive Ladungen anhäufen, oder an der Kathode Ionen von hoher Wertigkeit bald teilweise, bald völlig ihrer Ladung berauben.

Ein klassisches Beispiel hierfür bietet das von Bunsen erforschte Verhalten der Chromlösungen bei der Elektrolyse, für welches Ostwald ${ }^{13}$ ) in klarster Weise an der Hand der Ionentheorie eine sehr einfache Deutung im Sinne obiger Ausführungen gegeben hat. Ganz entsprechend werden in Eisenlösungen bei geringer Stromdichte an der Kathode Ferriionen zu Ferroionen (bei grösserer Dichte werden daneben zumeist Wasserstoffionen entladen), letztere an der Anode wieder zu Ferriionen gemacht. In fluorwasserstoffsaurer Lösung geht an der Kathode das fünfwertige Vanadium erst in das vierwertige, dann in das dreiwertige über, und Piccini und Giorgis ${ }^{14}$ ) konnten so die diesen Verbindungsstufen entsprechenden interessanten Doppelfluoride darstellen. Ähnlich gelangte $\mathrm{Piccini}{ }^{15}$ ) auch $\mathrm{zu}$ den Sulfatlösungen des dreiwertigen Vanadiums und Titans, aus denen er die betreffenden Alaune erhielt, während Marshall ${ }^{16}$ ) die Alaune des Kobalts und andere Kobaltiverbindngen darstellte, indem er bei niederer Temperatur an der Anode zweiwertige Kobaltionen in dreiwertige verwandelte. Ferner werden in Chlorid- wie Sulfatlösungen von zweiwertigem Kupfer an der Kathode neben

\footnotetext{
13) Allgemeine Chemie II, I, S. 995.

14) Gazz. Chim. 22, 1, 55 .

16) Journ. Chem. Soc. 9I, 760.

16) Gazz. Chim. 25, 2, 451 und 542 .
}

metallischem Kupfer einwertige Kupferionen erzeugt, welche an der Anode leicht die eine verlorene positive Ladung wieder aufnehmen, und ganz dieselben Erscheinungen bieten viele Quecksilberlösungen. Schliesslich sind derartige Vorgänge nicht auf Metallionen beschränkt, sondern treten auch an komplexen Ionen auf, so ist es bekannt, dass das Ion der Mangansaure $\overline{\mathrm{MnO}_{4}}$ unter Abgabe einer Ladungseinheit an der Anode in dasjenige der Übermangansäure $\mathrm{Mn}_{\overline{\mathrm{O}}}$ übergeht.

Man hat alle solche Vorgänge bisher zumeist anders erklärt: sie wurden nicht wie oben als primäre, sondern als sekundäre aufgefasst, veranlasst durch den an der Anode nascierenden Sauerstoff bezw. an der Kathode, soweit es sich um Metalle handelt, welche elektropositiver sind als Wasserstoff, durch den nascierenden Wasserstoff. Bei Eisenlösungen ${ }^{17}$ ) z. B. würde nach dieser Auffassung bei geringer Stromdichte der Wasserstoff ausreichen, das an der Kathode vorhandene Eisenoxydsalz in Eisenoxydulsalz zu verwandeln, während bei grösseren ein Teil von ihm unbenutzt entweicht, und entsprechend soll der Sauerstoff an der Anode Eisenoxydulverbindungen oxydieren oder bei grösseren Stromdichten zum Teil als Gas verloren gehen, ohne oxydierende Wirkung auszuüben.

Man sieht, diè ganze Angelegenheit lauft auf die von Le Blanc ${ }^{18}$ ) schon bei anderer Gelegenheit eingehend erörterte Frage hinaus, in wie weit man berechtigt ist, die Produkte der Elektrolyse als sekundär zu betrachten. Er zeigte, dass in sehr vielen Fällen die Annahme primärer Vorgänge die natürlichere und einfachere sei, und diese aus der Ionentheorie herausentwickelte Auffassung dürfte sich auch bei vielen Chemikern eingebürgert haben und ist wohl auch in der hier in Rede stehenden Frage die geeignetere, einheitlichere.

Stellt man den Satz auf, dass bei geringen Stromdichten an den Elektroden diejenigen Vorgange erfolgen, welche unter den gegebenen Bedingungen die geringste Arbeit erfordern, wăhrend bei höheren Stromdichten daneben auch Erscheinungen auftreten, zu denen grössere Arbeitsaufwände notwendig sind, so erlangt man in der That einen ausserordentlich einheitlichen Überblick über eine sehr grosse Anzahl elektrischer Vorgänge in allen

\footnotetext{
17) Vergl. Oettel, diese Zeitschr. I,go.

18) Lehrbuch der Elektroch. S. 210 u. ff.
} 
Einzelheiten. Die Änderung des Energieinhalts von Ionen eines und desselben Metalls dürfte in vielen Fallen geringere Arbeit erfordern als die Entladung von Wasserstoffionen, doch ist auf diesem Gebiete noch viel zu klären, am besten wohl durch das Studium von Oxydations- und Reduktionsketten. Es ist aber keineswegs ausgeschlossen, dass auch grössere Arbeitsaufwảnde, als sie zur Entladung von Wasserstoff - oder Hydroxylionen und zur Abscheidung der Gase gehören, zur Verảnderung der Ladungen mancher Ionen notwendig werden; alsdann erfolgt eine solche nur bei hoher Stromdichte. Beispielsweise hat $\mathrm{M}$ a rsh all die Kobaltiverbindungen unter den gleichen Versuchsbedingungen dargestellt wie überschwefelsaures Kali.

Eine sekundäre Entstehung vermittelst elektrolytischen Wasserstoffs kann man nun für Kupro- oder Merkurosalze aus Kupri- bezw. Merkurisalzen nicht annehmen, da Kupfer und Quecksilber elektronegativer sind als der Wasserstoff, só dass aus ihren Lösungen bei den zu den genannten Umsetzungen notwendigen niedrigen Stromdichten sicher kein Wasserstoff entionisiert werden kann.

In solchen Fällen hat man $z u$ einer anderen Erklärung gegriffen, indem man annimmt, dass der Strom aus Kupri- oder Merkurilösungen zunächst die dem Faradayschen Gesetz entsprechenden Metallmengen niederschlägt, von denen sich dann aber ein Teil, wie das Kupfer oder Quecksilber auch sonst unschwer thun, in der umgebenden Kupri - bezw. Merkurilösung unter Bildung von Kuprobezw. Merkurosalz wieder auflöst.

Also auch nach dieser Deutung würde es sich um einen sekundären Vorgang handeln, während die oben angeführte Auffassung, dass der Strom bei geringen Dichten Kupri- oder Merkuriionen nur die Hälfte ihrer Ladung nimmt, auch hier primäre Erscheinungen voraussetzt und diese also vom gleichen Standpunkt aus behandelt, wie die elektrolytischen Reduktionen und Oxydationen elektropositiverer Metalle.

Ich möchte jedoch meine Ansicht auch durch eine Beobachtung unterstützen: Wenn man eine angesăuerte Kuprisulfatlösung mit Kupferoxydul kocht, sie also mit dem nach Beobachtungen von O. Seidel und $\mathrm{mir}^{19}$ ) namentlich in der Hitze nicht allzu unbestăndigem Kuprosulfat săttigt, so scheidet diese Lösung, welche bei niederer Temperatur nun viel

19) Zeitschr. f. anorg. Chem. 14, 106. weniger Kuproionen zurückhalten kann, beim Erkalten metallisches Kupfer in schönen Krystallchen aus, nach der Gleichung

$$
2 \stackrel{+}{C}=\stackrel{+}{C} u+C u
$$

Wenn man nun bei gewöhnlicher Temperatur und bei sehr niedrigen Stromdichten, bei welchen nach meiner Annahme nur Kuproionen vom Strome erzeugt werden, angesäuerte Kupfersulfatlösungen elektrolysiert, so erhält man an einem als Kathode dienenden Platinblech stets das Kupfer in verstreuten, wohlausgebildeten Kryställchen, gleich als hätten diese sich aus einer elektrolytisch mit Kuproionen übersättigten Lösung abgeschieden. Bei I $0^{0}$, wo besonders reichlich in Sulfatlösungen Kuproionen entstehen, tritt diese Erscheinung selbst bei Stromdichten von I Amp./qdm noch auf. Würde das Kupfer erst in gewöhnlicher Weise vom Strome abgeschieden und löste sich dann zum Teil wieder auf, so wäre nicht einzusehen, warum das Kupfer nicht, wie sonst, als glatter einheitlicher Niederschlag auftreten sollte.

Wenn nun auf elektrolytischem Wege Salze bestimmter Verbindungsstufen der Metalle entstehen, so zeigen sie natürlich das gleiche chemische Verhalten, welches ihnen sonst eigentümlich ist. In neutraler Thalliumsulfatlösung nehmen, während an der Kathode 4 Thalloionen ihre Ladungen abgeben, an der Anode, wo dadurch $2 \mathrm{SO}_{4}^{-}$-ionen verfügbar geworden sind, zwei Thalloionen je zwei positive

$$
+++
$$

Ladungen auf, werden zu $T l$-ionen, d. h. es entsteht Thallisulfat in der Lösung. Dieses ist aber bekanntlich nur bei Gegenwart viel freier Säure in Lösung beständig, es erfährt also auch hier Hydrolyse, indem sich an der Anode braunes Thalliumsesquioxyd ausscheidet. Nur wenn man genügende Mengen freier Schwefelsäure der Lösung hinzufügt, unterbleibt die Hydrolyse und Thalliionen können sich in der Lösung halten.

Derartige Vorgänge sind indess keineswegs an die Anode gebunden. Auf einer ganz entsprechenden Hydrolyse des freilich für sich nicht zu untersuchenden Kuprosulfats beruht höchst wahrscheinlich auch die bei niederen Stromdichten an der Kathode zu beobachtende Abscheidung krystallisierten Kupferoxyduls bei der Elektrolyse von neutralen Sulfatlösungen. Säuert man diese an, so hindert die Sảure die Abscheidung von Kupferoxydul, ohne dass darum die Bildung von Kuproionen unterbleibt. 
Erscheint nun die Bildung des Bleisuperoxyds an der Anode nach der Le Blanc'schen Auffassung nicht als ein Einzelfall aus dieser ganzen grossen Reihe völlig einheitlich zu deutender Erscheinungen? Gewiss würde es schwer sein, für das Entstehen von Kupferoxydul oder Thalliumsesquioxyd im Sinne der Liebenow'schen Auffassung Anhaltspunkte für das Vorhandensein von $\mathrm{Cu}_{2} \mathrm{O}$ - oder $\mathrm{Tl}_{2} \mathrm{O}_{3}$-ionen in den betreffenden Sulfatlösungen zu gewinnen.

Freilich hat das Auftreten des Bleisuperoxyds aus salpetersaurer oder schwefelsaurer Lösung insofern etwas Besonderes, als überschüssige Säure seine Entstehung viel weniger als die der vorerwähnten Oxyde verhindert; nur aus sehr stark salpetersaurer. Lösung lässt sich Blei nicht mehr quantitativ als Superoxyd an der Anode fallen. Für essigsaure Lösungen hat andererseits, wie oben erwähnt, Elbs gezeigt, dass vor der Superoxydabscheidung an der Anode eine, wenn auch nur labile Lösung von Bleitetraacetat entsteht, und Bleitetrachlorid konnte ich, wie erwähnt, ganz leicht mit Hilfe der Elektrolyse darstellen. Die Unbestăndigkeit der meisten Salze des vierwertigen Bleis scheint mir nun aber kein hinreichender Grund zu sein, für die Erscheinungen der Elektrolyse der Bleilösungen eine von der Deutung anderer sonst aber ähnlicher Vorgänge ganz gesonderte Auffassung zu begründen.

Vielmehr können wir für die Leichtigkeit, mit der freie Säuren die Entstehung von Superoxyden an der Anode verhindern, die hierbei in Frage kommenden Metalle in eine Reihe ordnen, in welcher das Blei sich ungezwungen an die anderen anschliesst, und nur die letzte Stelle in der Reihe einnimmt. Hierbei muss darauf hingewiesen werden, dass die Neigung der Salze, Hydrolyse zu erleiden, sehr wesentlich mit abhängt von der Löslichkeit der dabei entstehenden Hydroxyde; je geringer diese ist, um so geringer muss auch durch vermehrten Saurezusatz die Zahl der $\mathrm{OH}$-ionen gemacht werden, damit das Hydroxyd in Lösung bleiben kann; dass aber die Löslichkeit der Hydroxyde das allein Bestimmende für die Beständigkeit von Salzen wäre, soll damit keineswegs gesagt sein. An den ersten Platz der erwähmten Reihe würde ${ }^{20}$ ) etwa das Thallium, an den zweiten das Wismuth zu stellen sein, alsdann, folgt das Mangan und schliesslich das Blei. In Classens

20) Von den nur bei grosser Verdunnung Hydrolyse erfahrenden Ferrilösungen sei abgesehen.
"Quantitative Analyse durch Elektrolyse" ist die Entstehung von Mangansuperoxyd an der Anode der des Bleisuperoxyds an die Seite gestellt, indem es auf das Vorhandensein von $\overline{\mathrm{MnO}}_{2}$-ionen zurückgeführt wird.

Nach der von mir vertretenen Ansicht ladet der Strom in einer Mangansalzlösung die Manganoionen an der Anode $\mathrm{zu} \stackrel{+++}{M_{n}}$ oder $\stackrel{++}{M n}$; die Salze dieser Verbindungsstufen des Mangans unterliegen bekanntlich sehr leicht der Hydrolyse, und so scheidet sich $M n(O H)_{3}$ oder $M n(O H)_{4}$ ab. Dieser Vorgang wird aber schon in reichlich schwefelsaurer oder salpetersaurer Lösung beeintrăchtigt bezw. verhindert; denn unter diesen Bedingungen lässt sich das Mangan nicht mehr quantitativ elektrolytisch als Superoxyd fällen; dessen Entstehung wird also erheblich leichter als die des Bleisuperoxyds durch freie Săure verhindert.

Ähnlich diesem vermag endlich auch das Mangansuperoxyd in galvanischen Elementen, z. B. dem Leclanché'schen, gegenüber einem geeigneten Metall, hier dem Zink, sich elektromotorisch zu bethätigen. Auch diesen Vorgang hat Le Blanc ${ }^{21}$ ) auf die Entstehung vierwertiger Manganionen und ihren leichten Übergang zur Zweiwertigkeit in befriedigender Weise zurückführen können. ${ }^{22}$ )

Nach allem erscheint die Le Blanc'sche Auffassung der Vorgănge an der Anode des Bleisammlers in guter Übereinstimmung mit einer unter den ihr entsprechenden Gesichtspunkten völlig einheitlich darzustellenden, grossen Erscheinungsreihe. Hierbei darf jedoch nicht unerwăhnt bleiben, dass Liebenow am Schluss seiner zusammen mit Strasser ausgeführten schönen Arbeit über das Kohlenelement ${ }^{23}$ ) versucht, auch seine Betrachtungsweise

21) Lehrbuch f. Elektroch. S. 221.

22) Die über das Potential solcher "Superoxydelektroden" von Tower und von Smith (Zeitschr. f. physik. Ch. 18, 17 u. 2I, 93) im Wesentlichen auf Grundlage der Le Blancschen -Anschauung unternommenen Versuche haben dies ausreichend bestätigt. Man würde aber auch nach der Liebenow'schen Theorie ziemlich zum gleichen Ergebnis gekommen sein, da danach z. B. die $\overline{M n}_{\overline{2}}$-ionen in der Lösung sehr bald zerfallen im Sinne der Gleichung: $\overrightarrow{\mathrm{Mn}_{\mathrm{O}}}+4 \stackrel{+}{\mathrm{H}}+{ }_{4} \overrightarrow{\mathrm{OH}}$ $=\stackrel{+}{\mathrm{M} n}+{ }_{4} \mathrm{H}_{2} \mathrm{O}+\mathrm{O}_{2}$; es wäre also auch in diesem Falle das Potential abbängig von der Konzentration der Manganoionen sowie der vierten Potenz derjenigen der Wasserstoffbezw. der Hydroxylionen.

29) Diese Zeitschr. 3, 353. 
jener Dinge an weitere Erscheinungskreise anzuschliessen, und zwar setzt er die Entstehung von $\overline{P b} \overline{O_{z}}$-ionen aus Bleisuperoxyd in Beziehung zu dem Passivwerden gewisser Metalle. Dieser $\mathrm{Zu}$ sammenhang erscheint aber vor der Hand doch noch nicht ganz gesichert, so dass es der Zukunft überlassen bleiben muss, zu zeigen, ob diese von Liebenow vorausgesehenen Beziehungen wirklich existieren.

Die meisten der von mir oben angeführten Erscheinungen sind erst qualitativ beobachtet und bedürfen vielfach noch der quantitativen Durcharbeitung, wobei sich vielleicht weitere Anhaltspunkte für die von mir gezogenen Analogieschlüsse ergeben werden. Aber selbst wenn das Gegentheil hiervon eintreten sollte, so hätten meine Ausführungen doch auch dann ihren $Z$ weck erreicht, wenn sie dazu angeregt hätten, bei der Erforschung der so wichtigen und interessanten Erscheinungen im Bleisammler nicht ausschliesslich die Elektrolyse der Bleilösungen im Auge zu behalten, sondern sie, soweit es die Natur der Sache erlaubt, unter weiteren Gesichtspunkten mit anderen ăhnlichen Vorgängen zu vergleichen, welche oftmals gewisse Dinge klarer erkennen lassen, als es gerade die Eigenart des Bleisammlers zulässt.

Dresden, 20. April I 897.

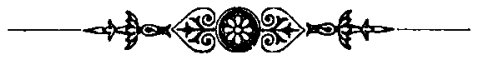

\section{REPERTORIUM.}

\section{STROMERZEUGUNG.}

Element von Th. F. Boland, Ch. J. Hubbell und H. C. Hubbell (Engl. Pat. Nr. 20077 von I896) zum Beleuchten von Fahrrädern u. dergl. (Fig. 483 bis 488 .) In dem Gefässe $I$ sind drei Zink-Kohle-Elemente und gleichzeitig eine Glüh-

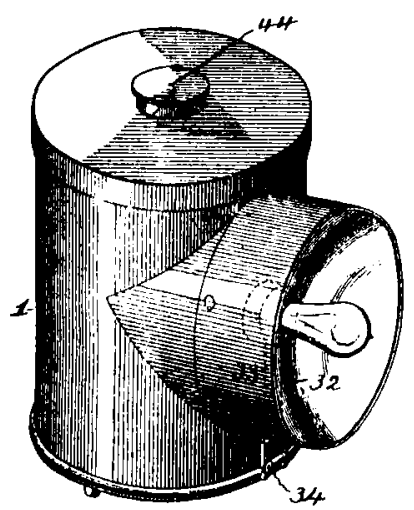

Fig. 483 .

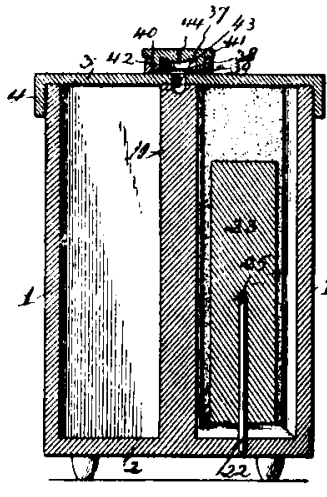

Fig. $4^{85}$.

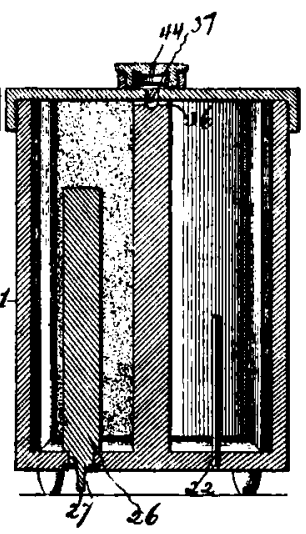

Fig. 486 .
Durch die Schlitze $3^{6}$, Loch 37 und 44 können die im Elemente sich entwickelnden Gase entweichen. Alle Kohlen- bezw. Zinkpole sind unter sich verbunden und führen zu den Klemmen 34, von wo die Drăhte 33 und $3^{2}$ nach der Lampe gehen.

Ee.

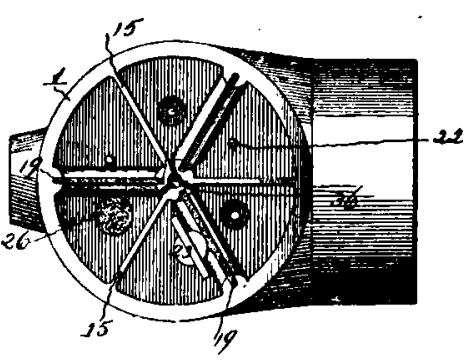

Fig. 484 .

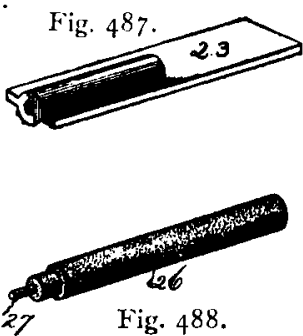

lampe mit Reflektor enthalten. Durch die Rippen 15 ist das Gefäss in drei Abteilungen getrennt und jede dieser Abteilungen wieder in zwei Kammern durch poröse Platten ig. In drei von diesen sechs Kammern sind Zinkelektroden 23, wảhrend in den übrigen drei die Kohlenpole 26 sich befinden. Sämtliche Pole endigen im unteren Boden und zwar die Zinkpole bei 22, die Kohlenpole bei 27 .
Elektrische Batterie von W. Turner (Engl. Pat. Nr. I669 von I 896). (Fig. 489 und 490). Die Batterie, bestehend aus einer grösseren Anzahl von Elementen, wird in dem Raume $F$ untergebracht. Über diesem Raume befinden sich noch die beiden Kammern $B$ und $D$; beide haben im Boden Ventilchen $C_{1}$ und $E_{1}$. Der von der Batterie erzeugte Strom treibt einen Elektromotor $K$, und dieser durch 